\title{
ENHANCEMENT OF CARMAC PROTOCOL FOR MULTICHANNEL WITH DIRECTIONAL ANTENNAS IN WIRELESS IOT NETWORKS
}

\author{
Gaurav Aggarwal
}

Department of Computer Science \& Engineering

Ramgarhia institute of Engineering \&Technology,

Punjab Technical University Kapurthala, Punjab

\begin{abstract}
In this paper discussed The Internet of things (IoT) express physical devices that are set with sensor nodes, working capability, and other technologies that anchor and sharing data with other devices and systems over the internet. The nodes do not use any infrastructure for communication, such as a base station and wired access point. A critical problem arises in wireless sensor networks is power consumption. Another problem arises in wireless sensor networks is hidden terminal and Exposed terminal problem which reduced the throughput and increases End to End delay .To solve the problems used Directional antennas which is enhancing the performance of CARMAC Protocol by imposing transmission and receiving GPS signals on the wireless sensor nodes. In proposed work increases the sectors of the directional antennas and can be used to solve hidden terminal and exposed terminal problem by using ECARMAC protocol for wireless sensor network.
\end{abstract}

Keywords: ECARMAC, Hidden terminal, Exposed terminal,

Deafness, Directional antenna

Introduction of wireless IoT network- The Internet of things (IoT) express physical devices that are set with sensor nodes, working capability, software, and other technologies that anchor and sharing data with other devices and systems over the Internet. Traditional fields of embedded systems, wireless sensor networks, control systems, including home and building automation, independently and collectively enable the Internet of things. The term "Things" in the Internet of Things refers to anything and everything in day to day life which is accessed or connected through the internet. As Show in Fig 1.
Er Parminder Singh

HOD of Department of Computer Science \& Engineering ,

Ramgarhia institute of Engineering \&Technology,

Punjab Technical University Kapurthala, Punjab

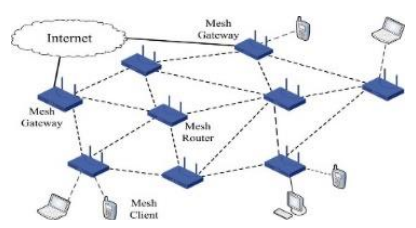

Fig 1. wireless IoT network

\section{Applications of wireless IoT networks}

There are various Fields were IoT Networks are used:

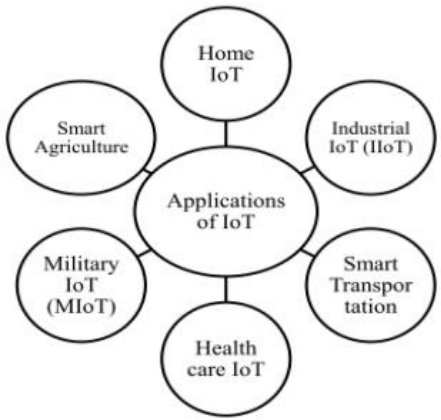

Fig 2. Application of Wireless IoT networks

Home IoT: Home IoT consists of three components such as server, network gateway and smart devices. Smart devices are sensors embedded with $\mathrm{TV}$, or Sensors embedded with Doors.

Industrial IoT: IoT in industries lead to Industrial Internet of Things (IoT) which has added more value to industry. Sensor and actuators are used in industrial environment to improve manufacturing process intern to reduce the cost, to increase efficiency of providing services to customer. 
Military IoT: IoT concept is introduced into Military domain by integrating sensors to military things for the purpose of military, formed Internet of Military Things (IoMT) or military internet of Things (MIOT). In IoMT Sensors or actuators, RFID are embedded to military equipment or within the military information infrastructure information such as location, Threat recognition, attacker movement etc. This information helps proper utilization of resources and efficient military operation.

Health care IoT: Wearable devices are small in size and sensors are embedded within it.These wearable are placed in end user or patient, which sense physiological data and movement. By using communication technology such as Bluetooth or Wi-Fi or $3 \mathrm{G}$ or $4 \mathrm{G}$, these data will be sent to remote data centre. By using data analysis techniques, these data will be analysed and clinically relevant information will be extracted. action to take on plant such as sprinkling water, switching on fan, curtain control to control heat from direct sun light etc. With this it reduces human resources and saves energy.

Agriculture IoT: There are numerous IoT applications in farming such as collecting data on temperature, rainfall, humidity, wind speed, pest infestation, and soil content. This data can be used to automate farming techniques, take informed decisions to improve quality and quantity, minimize risk and waste, and reduce the effort required to manage crops.

\section{Major issues in wireless IoT network \\ 1. Hidden terminal problem \\ 2. Exposed terminal problem}

\section{Hidden Terminal problem}

Hidden nodes are the nodes that are not in the range of other nodes or a group of nodes. In wireless networking the Hidden Node problem occurs when a node is visible from wireless Access point(AP),but not from other nodes communicating with said AP. Hidden node problem is one of the major problems which leads to packet dropping and transfer delays. Example: In hidden Node scenario consists of three nodes A,B and C. In this case Node A can hear Node B, but not Node
$\mathrm{C}$, as it is not in the same transmission range. Each Node that is located in the other node's transmission range can receive its packet easily. Therefore in Fig 3.

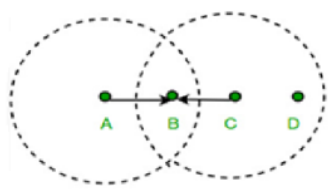

Fig 3. Hidden terminal problem

Node B can receive packets from both Nodes A and C. However, there will be collision at Node B if these two nodes send their packets at same time, and Node B cannot successfully receive any packets. Hidden Node problem effects on power consumption transmission delay, nodes efficiency and QoS.

\section{Exposed Terminal Problem}

In wireless networks, the Exposed Node Problem occurs when a node is prevented from sending packets to other nodes due to a neighboring transmitter. Consider an Example of 4 nodes labeled A, B, C, and D, where the two receivers are out of range of each other, yet the two transmitters in the middle are in range of each other.

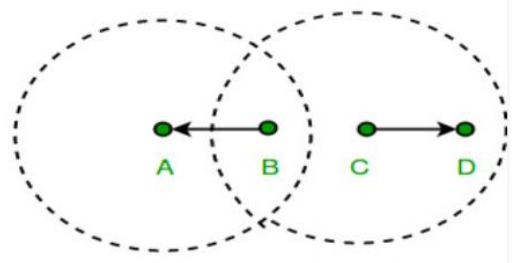

Fig 4. Exposed terminal problem

as shown in Figure 4. Suppose B is sending to A. Node $\mathrm{C}$ is aware of this communication because it hears B's transmission. It would be a mistake for $\mathrm{C}$ to conclude that it cannot transmit to anyone just because it can hear B's transmission. suppose C wants to transmit to node $\mathrm{D}$. This is not a problem since C's transmission to $\mathrm{D}$ will not interfere with A's ability to receive from B.

maxima are positioned as shown.

\section{Basic antenna parameters}

1. Radiation pattern: - The radiation pattern of an antenna is a plot of the far-field radiation from the 
antenna. More specifically, it is a plot of the power radiated from an antenna per unit solid angle, or its radiation intensity $U$. This is arrived at by simply multiplying the power density at a given distance by the square of the distance $r$, where the power density $S$ is given by the magnitude of the time-averaged Poynting vector: $\quad U=r^{2} S$

2. Directivity: - The directivity of an antenna, a function of direction is defined by the ratio of radiation intensity of an antenna in the direction to the mean radiation intensity in all directions.

3. Power Gain:- The power gain or simply the gain, of an antenna is the ratio of its radiation intensity to that of an isotropic antenna radiating the same total power as accepted by the real antenna. When antenna manufacturers specify simply the gain of an antenna they are usually referring to the maximum value of gain. 4. Bandwidth:- The bandwidth of an antenna expresses its ability to operate over a wide frequency range. It is often defined as the range over which the power gain is maintained to within $3 \mathrm{~dB}$ of its maximum value. The radiation pattern of an antenna may change dramatically outside its specified operating bandwidth.

5.Beamwidth:- Beamwidth describes the angular aperture where the most important part of the power is radiated.

\section{Antenna Model}

In Enhanced CARMAC, it is assumed that a single radio transceiver is associated with each IoT-enabled wireless node and operations are made in full-duplex mode. Hence, during communication, it can either transmit or receive electromagnetic radio signals. It is also assumed that the radio transceiver able to switch among a group of independent frequency channels.

Directional Coverage Area:- To cover all directions, we use $S^{*} B>2 \pi$, where $S$ is the number of antenna sectors and $B$ is beamwidth of the single antenna sector. Data packets are transferred using one sector .

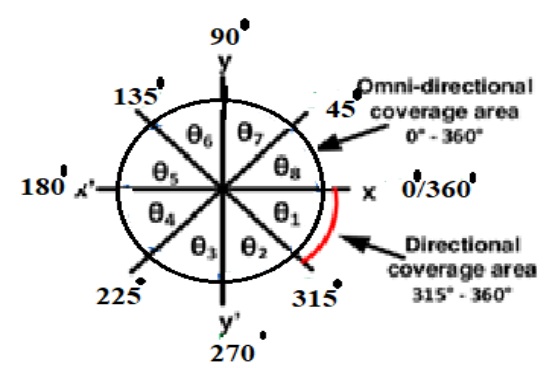

Fig 5. Antenna Model

GPS Enabled node:- Since, each IoT node is equipped with Global Positioning System (GPS), IoT nodes are capable of determining the accurate location of its own and overhear the location of all neighboring nodes through control frames. Using GPS coordinate information a node calculates the distance and directional position of each of its neighbor nodes from its own.

\section{Review of Literature}

In this work brief explanation of the previous inspect made by different researchers related to this research. There are several MAC protocols proposed over the time, out of them few are discussed below to understand the issues that have been faced while developing such techniques.

In this paper, they proposed a Cooperation-based Adaptive and Reliable MAC (CAR MAC) Design for Multichannel Directional Wireless IoT Networks that combines both of these cooperation and multichannel directional concepts of cooperation. Multichannel directional hidden terminal problems and deafness problems in medium access are solved using both concepts of cooperation jointly. Besides, multidirectional data packet relaying in the same data channel enables parallel transmission that increases the bandwidth utilization. Moreover, the proposed protocol uses a smart GPS based neighbor discovery. Therefore, the directional position and distance among the IoTenabled wireless nodes are smartly determined to make the control channel cooperation more informative. The results of extensive simulations reveal that CAR MAC 
achieves significant improvement in network performances.

In this paper, it has been witnessed that wireless systems based on IoT-based have developed rapidly in various sectors. The IoT is the network in which physical devices, equipment, sensors and other objects can communicate among themselves without human involvement. The WSN is a central component of the IoT, which has proliferated into several different applications in real-time. The IoT and WSNs now have various critical and non-critical applications impacting nearly every area of our everyday life. WSN nodes are usually small and battery-driven machines. Thus, the energy effective data aggregation techniques that increase the lifespan of the network are highly significant. Various approaches and algorithms for energy-efficient data aggregation in IoT-WSN systems were presented. This paper reviews the literature with specific attention to aspects of wireless networking for the preservation of energy and aggregation of data.

The IoT based power management system requires data from the feeder in the grid. Sufficient power supply with demand is a significant challenge for several challenges for several countries around the world. Rapid growing demand for power supply requires power quality enhancement to get higher reliability in the smart grid. This smart power system sensor equipped that measures the grid power capacity and update to the organization on a consistent schedule. Energy supplies to specific region indicated by power install capacity in the grid use a global system for mobile communication messaging service to notify customers of power generation and power supply time . the IoT based wireless sensor network is revolutionary system for smart monitoring . In this paper proposed a system demonstrate for progress and implementation of wireless sensor based communication system for smart maintain power quality. The dynamic controller has controlled the event of power quality problem and voltage rise. Appropriate systems and controllers have been demonstrated and analyzed for control performance of a monitoring system in the smart grid.

\section{Performance Evolution}

The performance of our proposed ECARMAC protocol is evaluated using simulation results. The simulation models of our proposed protocol along with the compared protocols are designed and developed in NS2 simulator. NS2 is an open-source event-driven simulator designed specifically for research in computer communication networks. NS2 contains modules for numerous network components such as routing, transport layer protocol, and application. To investigate network performance, researchers can simply use an easy-to-use scripting language to configure a network, and observe results generated by NS2 We compared the simulation results of the proposed ECARMAC with present CARMAC on the bases of throughput, end-toend delay and collision probabilty. Therefore, the simulation results of ECAR MAC are compared with CARMAC.

\begin{tabular}{|l|l|}
\hline \multicolumn{2}{|c|}{ Simulation Parameters } \\
\hline Parameters & Value \\
\hline Simulation Area & $1000^{*} 1000 \mathrm{~m}^{2}$ \\
\hline Node Deployment transmission & $400 \mathrm{~m}$ \\
\hline Omnidirectional Uniform Random & \\
\hline Directional Transmission Range & $825 \mathrm{~m}$ \\
\hline Number of Data Channel & 5 \\
\hline No of control channel & 1 \\
\hline RTS/CTS/MCTS length & 25 byte \\
\hline VTR/CTR Length & 31 byte \\
\hline Data packet size & 512 byte \\
\hline Ack length & 5 byte \\
\hline Number of sector & $4-10$ \\
\hline No of simulation run & 10 \\
\hline
\end{tabular}

\section{Simulation Result of Enhanced Cooperation Based Adaptive and Reliable MAC (ECARMAC) Protocol}

In this scenario, topology creation of wireless IoT networks with randomly distributed nodes in area $1000 * 1000$ and the transmission of the packets between the nodes is done using AODV routing protocol. First, we find the Collision Probability. Secondly we find the performance of the network on the basis of End to End delay and throughput.

\section{Simulation Results}

In this research nodes are randomly deployed and they are transmitting data packets from one node to another directionally. 
Collision probability- shows a Collision probability comparison of ECARMAC protocol and CARMAC protocol

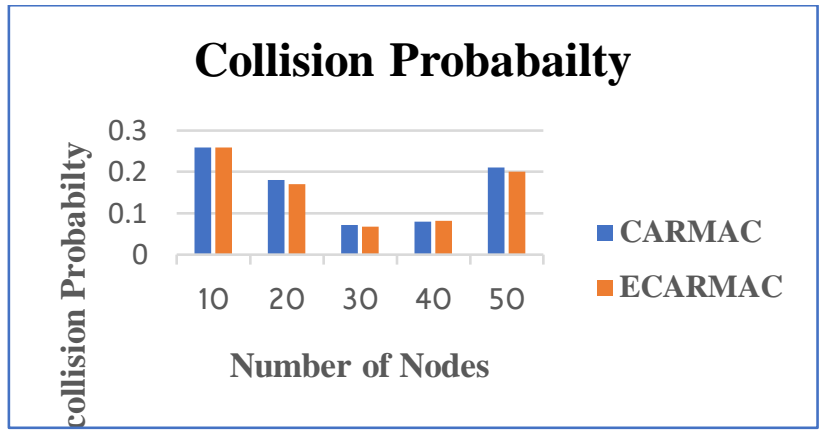

Figure 6 Comparison of Collision probability

\section{End to End Delay}

End to End delay comparison of ECARMAC protocol and CARMAC protocol as in graph.

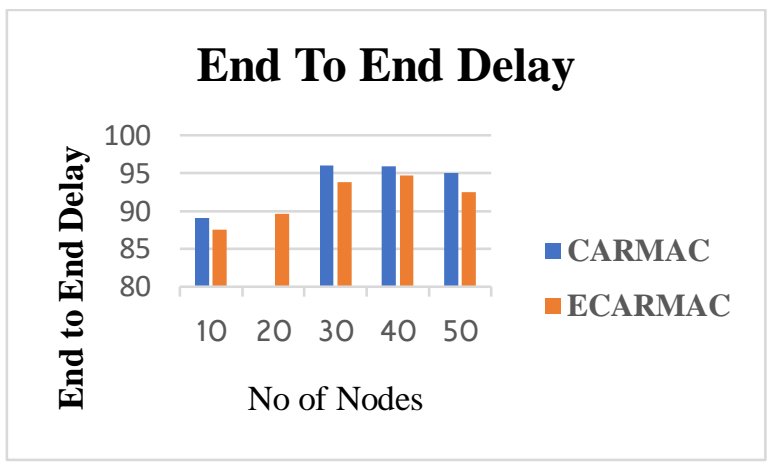

Figure 7. Comparison of End To End Delay

\section{Throughput}

In the following section we compare the throughput of the Enhanced CARMAC protocol with CARMAC protocol for different nodes. protocol has better performance than CARMAC protocol.

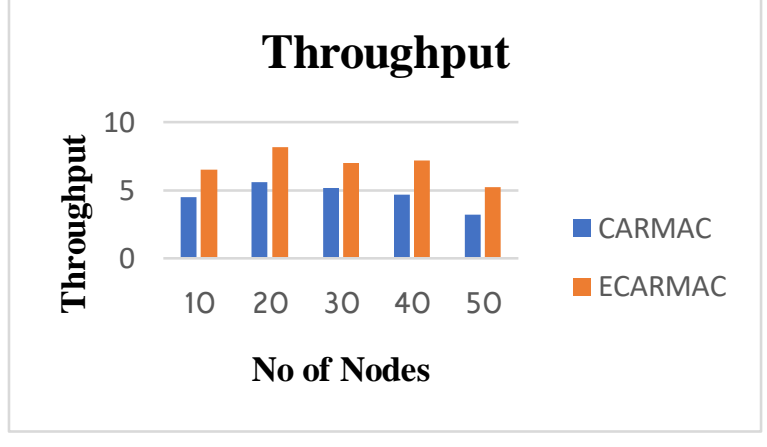

Figure 8 Comparison of Throughput

\section{Conclusion}

The wireless IoT system is express physical devices that are set with sensor nodes, working capability, software, and other technologies that anchor and sharing data with other devices and systems over the Internet. Due to node there are various problems occurred in the network. Hidden terminal and Exposed terminal problems are major problems which reduces the performance of the network. In this study investigated various MAC protocols Problem. CARMAC is cooperative based adaptive and reliable MAC protocol which enabled GPS system when nodes communicate. In the base paper, the enhancement in CARMAC protocol is proposed work with increased sector of directional antenna in case of improvement the performance of the wireless IoT network. In this research work, CARMAC protocol will be improved and increases the performance of the network and reduces the hidden and exposed terminal problems which causes collision on the network. The proposed improvement brings on to decreases end to end delay, increases throughput and resolve collision problem.

\section{Reference}

[1] Md. tareq Mahmud, Md. obaidur rahman, salman a. alqahtani, and mohammad mehedi hassan," cooperation-based adaptive and reliable mac design for multichannel directional wireless iot networks"(ieee), volume 9, pp 97518-97538,2021.

[2] Kamal gulati, raja sarath kumar boddu, dhiraj kapila, sunil 1. bangare , neeraj chandnani . saravanan," a review paper on wireless sensor network techniques in internet of things (iot)(researchgate)pp1-5, 2021.

[3] Amam hossian bagdadee, md zahirul hoque , li zhang,"iot based wireless sensor network for power quality control in smart grid"sciencedirect, pp 1148-1160,2020.

[4] Mahdi zareei, a. k. m. muzahidul, sabariah baharun, cesar vargasrosales, leyre azpilicueta , nafees mansoor" medium access control 
protocols for cognitive radio ad hoc networks: a survey",pp 1 $19,2017$.

[5] Satbir singh, rydhm beri" hidden node problem in wireless adhoc network", advances in computational sciences and technology, volume 10 ,pp 1819-1824,2017.

[6] Shruti g hegde, "internet of things (iot): a study on architectural elements, communication technologies and applications", (ijarcce)international journal of advanced research in computer and communication engineering, volume 5,issue 9,pp 189-193, 2016.

[7] Crissi mariam robert, dhanya s," addressing hidden terminal problem in heterogeneous wireless networks", international journal of engineering research \& technology (ijert), volume 4,issue 09,2015 .

[8] Mario manzano, felipe espinosa, ángel m. bravo-santos, alfredo gardel-vicente," cognitive self-scheduled mechanism for access control in noisy vehicular ad hoc networks", volume 2015, article id 354292, pages-12. 